\title{
The Heimlich Valve for Pleural Cavity Drainage
}

Winston Ominde Makanga ${ }^{1}$, Andrew Nyaoncha Nyangau ${ }^{2}$, Benjamin Njoga Njihia ${ }^{3}$

1. St Mary's Mission Hospital, Elementaita

2. St Mary's Hospital, Nairobi

3. Aga Khan University Hospital, Nairobi

Correspondence to: Dr. Benjamin Njoga Njihia, P.0 Box 51982-00200 Nairobi. Email: benjamin.njihia@gmail.com

\begin{abstract}
Introduction: Traditional chest tube fixation and drainage has been undertaken using standard rigid chest tubes connected to under water seal bottles. These are bulky, cumbersome, expensive, and pose a risk of accidental air suction into the chest. One-way valve systems such as the Heimlich valve are small, portable apparatus that allow regulation of fluid flow and require minimal nursing care other than daily charting. Methods: A retrospective descriptive analysis of all chest drains connected to a Heimlich valve between January 2009 and December 2012. Data on indications, duration of drainage and frequency of complications was collected. Results: Fifty seven chest
\end{abstract}

\section{Introduction}

Pleural effusion develops in disease states that lead to altered dynamics of pleural fluid turnover (1). A common clinical presentation in symptomatic patients is difficulty in breathing with variable signs (2). In the Unites States, about $12 \%$ of patients with pleural effusions will undergo thoracentesis and historically, improvements in oxygenation, pulmonary mechanics and gas exchange have been cited $(3,4)$. More recent work has also demonstrated that thoracentesis confers patients with better re-adaptation to daily activities (5).

Various methods for venting the pleural cavity include a thoracic catheter attached to an underwater seal drainage system or a one-way valve system such as the Heimlich valve $(6,7)$. These have been shown to be equi-efficacious in draining the pleural cavity (8-10). However, underwater seal drainage systems have been shown to have poor tolerability from the patients' perspective (11) while the one-way valves allow for outpatient management of pleural drainage (12-14). Although classically described for pneumothoraces, the characteristics of the Heimlich valve allow it to be used for draining fluid as well (10). It is anecdotally known that underwater seal drainage systems are the preferred modality of pleural tubes connected to a Heimlich valve were inserted over the study period. Majority were for pleural effusions. No complications were encountered. Four patients (7\%) required thoracotomy. The average duration to removal was 6 days and all patients reported satisfactory comfort and mobility. Conclusion: The Heimlich valve is a feasible and cheap alternative method of chest tube drainage with high rates of success and very low morbidity. We propose their usage in resource-constrained settings.

Keywords: Thoracentesis, Heimlich, Thoracostomy

Ann Afr Surg. 2016;13(2):45-8.

DOI: http://dx.doi.org/10.4314/aas.v13i2.2

drainage in Kenya. However, shortcomings such as understaffing and prolonged hospital stay make the case for consideration of alternative ways to drain the pleural cavity $(15,16)$.

The Heimlich chest drainage valve was developed to allow for safe, simple, and efficient drainage the pleural cavity. The Heimlich valve connects to chest tubing and allows fluid and air to pass in one direction only thus can replace the cumbersome underwater drainage bottle system. The valve, which functions in any position, need never be clamped, and regulated suction can be attached to it if necessary. The valve drains into a plastic bag that can be held at any level, allowing the patient to be ambulatory by carrying the bag. The construction and function of the valve is easily understood by medical and nursing staff. It is pre sterilized, stored in a sterile package, and readily utilized on emergency vehicles and in the operating room $(17,18)$. This paper shares our experience with the Heimlich valve in the management of both pneumothoraces and pleural effusions, with a review of the literature.

\section{Methods}

The retrospective study was conducted at the St Mary's Mission Hospital, Elementaita over a 4-year 
period (January 2009 to December 2012). All chest tube insertions were performed by a qualified general surgeon using an aseptic technique under local anesthesia. Where the procedure was not tolerable or the patient was too young to co-operate, additional sedation was used. Following insertion, all chest tubes were connected to a one way Heimlich valve which was in turn connected to a urine bag. The pneumothoraces were connected to a Heimlich valve and to a urine bag that was fenestrated to allow for decompression of expelled gas. All chest tubes inserted had radiographic confirmation of correct placement.

All patients remained inpatients and were followed up until tube removal. Daily charting of the amount of effusion drained was done by the ward nurse. The patients were allowed to ambulate guided by their general health condition. The criteria for removal of a chest drain as per institutional protocol were: 24-hourly drainage of less than $100 \mathrm{ml}$ for patients with pleural effusion or hemothorax; complete resolution of drainage for empyema; and auscultation of satisfactory breath sounds for pneumothorax. Before removal of the chest tube, radiographic confirmation of resolution was obtained by a chest radiograph.

Data were extracted from patients records detailing duration to resolution, indication for chest tube insertion, complications (pneumothorax, accidental dislodgement, blockage and malfunction), and requirement for additional therapeutic intervention. Data were summarized as frequencies, percentages and means.

\section{Results}

In the study period,57 patients had chest tubesinserted. Of these, $40(70 \%)$ were inserted for pleural effusion, $8(14 \%)$ for pneumothorax, $6(11 \%)$ for empyema thoracis and $3(5 \%)$ for traumatic hemothorax. The average age was 34.4 years (7-86yrs); there were 35 male and 22 female patients. Two of the patients were below 18 years.

There were no complications reported during insertion of chest tubes. On average it took six days to achieve full lung re-expansion. None of the inserted tubes and drainage systems developed complication i.e. re-accumulation, pneumothorax, tube infection, accidental disconnection with attendant pneumothorax. Four of the patients with empyema required a subsequent thoracotomy after a subsequent chest radiographs showed loculated effusion with collapsed lung and thickened visceral pleura. All patients were sufficiently mobile immediately postinsertion.

\section{Discussion}

This paper summarizes data from a centre which routinely uses Heimlich-valve-based drain system as opposed to underwater seal drains that are more popular in hospitals in the country. A Heimlich valve (flutter valve) is a specially designed assembly comprising of a sealed transparent housing and a valve that establishes a unidirectional flow path for air or fluid (Figure 1). This assembly is equipped with tubing connection ends which are marked differently to identify the inflow and outflow ends (blue at the chest end and clear at the reservoir end). The Heimlich valve is eponymously named after its inventor (18). Since its introduction, the Heimlich valve has been advanced as an alternative method to more traditional methods such as the underwater seal for chest drainage (Figure 2). Several studies have demonstrated the Heimlich valve to be equi-efficacious to underwater seal drainage (8-10). This method has also been used in the ambulatory setting for chest drainage, especially where it is required for a prolonged duration of time. There are several advantages of the Heimlich valve over the traditional under-water seal drainage.
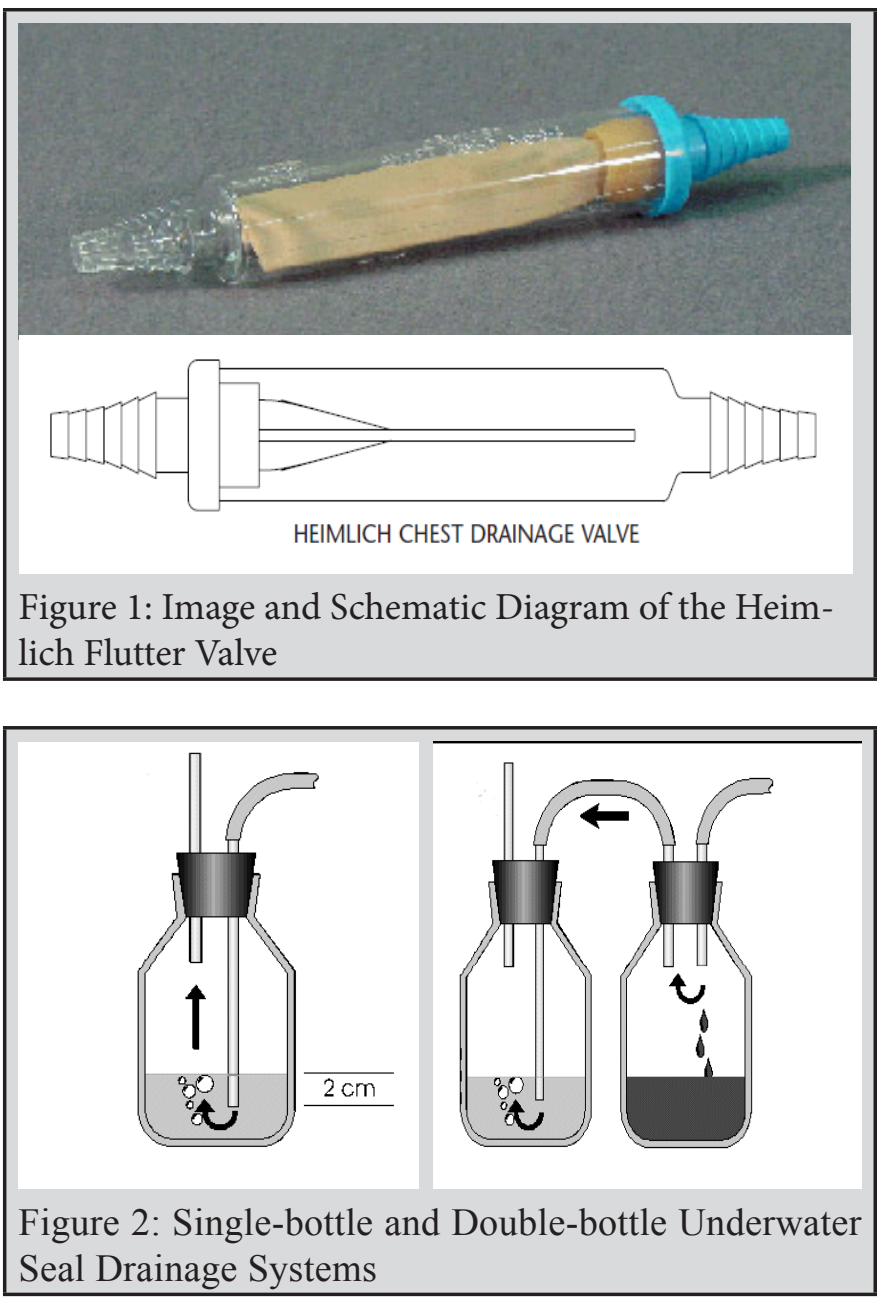

It is easy to use: it comes readily assembled and requires only to be connected in the right orientation to a thoracostomy tube. There is no need to connect 
it to any amount of fluid to establish a seal as in the underwater seal drainage. This also averts the confusion that may arise when reading off the amount of drainage as any amounts collected can only be from the patient. One other major disadvantage of the underwater seal drainage is that chest drainage is significantly affected when the water column creating the seal is higher than $2 \mathrm{~cm}$, as would happen once fluid begins to fill the bottle system (Figure 2). This leads to an inconsistent drainage unless the bottle is closely monitored and promptly emptied. Similarly, the observed reduction of fluid level can be erroneously due to a high fluid column impeding further drainage as opposed to actual reduction in the amount of effluent. This can be mitigated by an additional bottle connected to suction, a system even more cumbersome than the single bottle system. The Heimlich valve averts this demerit while at the same time allowing for safe connection to suction to enhance drainage (8).

There is no need for clamping the tubes to empty and drain or change the collecting system. This makes the assembly less cumbersome, and if properly trained, the measurement and drainage can be carried out sufficiently safely by the patient. The Heimlich valve system does not restrict the patient to the bed. This allows for patients who may require prolonged drainage to be nursed in the outpatient setting while being sufficiently independent. Additionally, there is no danger of the drained effluent or air flowing back into the pleural cavity with assumption of a different or dependent position relative to the collecting bag. With a well secured thoracostomy tube, the patient enjoys relative freedom in terms of nursing positions (10).

The cost of each Heimlich valve is 300 shillings (USD 3) compared to 3,500 shillings (USD35) for the underwater seal drainage system. These valves are packaged as single use disposable supplies. The traditional underwater seal drainage consists of reusable sterilizable glass bottles and rubber tubing. These are not only expensive, but often are unavailable whenever an institution has more patients requiring chest drainage than the available units. All these demerits are readily mitigated by the Heimlich valve. The safety profile of the Heimlich valve has been shown to be good with rare complications (19). These results establish that the Heimlich valve is an effective option for use during chest tube drainage with very low rates of morbidity and with satisfactory outcome. The indication for thoracotomy in four of the patients was loculated empyema and these are unlikely to have been delimited by use of an alternative method of drainage. Furthermore our patients mostly required minimal nursing care of their drains: emptying drainage bags and charting the volumes. None of our patients received chest drainage in the ambulatory or out-patient setting as this was not required.

\section{Conclusion}

The Heimlich valve is a feasible and cheap alternative method of chest tube drainage with high rates of success and very low morbidity. It is especially useful in a setting of high patient to nursing staff ratio. We propose it for adoption in local institutions over the underwater seal for chest drainage as well as trials that will offer greater insight as to patient perspective and cost comparison.

\section{References}

1. Miserocchi G. Mechanisms Controlling the Volume of Pleural Fluid and Extravascular Lung Water. Eur Respir Rev. 2009;18(114):244-52.

2. Kalantri S, Joshi R, Lokhande T, et al. Accuracy and Reliability of Physical Signs in the Diagnosis of Pleural Effusion. Respir Med. 2007;101(3):431-8.

3. Agustí AGN, Cardús J, Roca J, et al. VentilationPerfusion Mismatch in Patients with Pleural Effusion: Effects of Thoracentesis. Am J Respir Crit Care Med. 1997;156(4 pt 1):1205-9.

4. Wang JS, Tseng $\mathrm{CH}$. Changes in Pulmonary Mechanics And Gas Exchange after Thoracentesis on Patients with Inversion of a Hemidiaphragm Secondary to Large Pleural Effusion. Chest. 1995;107(6):1610-4.

5. CartaxoAM,VargasFS,SalgeJM, etal.Improvements in the 6-min Walk Test and Spirometry Following Thoracentesis for Symptomatic Pleural Effusions. Chest. 2011;139(6):1424-9.

6. Repanshek ZD, Ufberg JW, Vilke GM, et al. Alternative Treatments of Pneumothorax. J Emerg Med. 2013;44(2):457-66.

7. Lodi R, Stefani A. A New Portable Chest Drainage Device. Ann Thorac Surg. 2000;69(4):998-1001.

8. Vuorisalo S, Aarnio P, Hannukainen J. Comparison Between Flutter Valve Drainage Bag and Underwater Seal Device for Pleural Drainage after Lung Surgery. Scand J Surg. 2005;94(1):56-8.

9. Waller DA, Edwards JG, Rajesh PB. A Physiological Comparison of Flutter Valve Drainage Bags and Underwater Seal Systems for Postoperative Air Leaks. Thorax. 1999;54(5):442-3.

10. Graham AN, Cosgrove AP, Gibbons JR, et al. Randomised Clinical Trial of Chest Drainage Systems. Thorax. $1992 ; 47(6): 461-2$.

11. Owen S, Gould D. Underwater Seal Chest Drains: The Patient's Experience. J Clin Nurs. 1997;6(3):215-25.

12. Hassani B, Foote J, Borgundvaag B. Outpatient 
Management of Primary Spontaneous Pneumothorax in the Emergency Department of a Community Hospital Using a Small-Bore Catheter and a Heimlich Valve. Acad Emerg Med. 2009;16(6):513-8.

13. Chan KY, Fikri-Abdullah M, Sajjad M, et al. Outpatient Treatment of Spontaneous Pneumothorax Using an Improved Pocket Sized Heimlich Valve. Med J Malaysia. 2003;58(4):597-9.

14. Vuorisalo S. Flutter Valve Drainage Bag is a Useful Device for the Pleural Drainage. Scand J Surg. 2001;90(4):294-6.

15. Briggs D. Nursing Care and Management of Patients with Intrapleural Drains. Nurs Stand.
2010;24:47-55.

16. Durai R, Hoque H, Davies TW. Managing a Chest Tube and Drainage System. AORN J. 2010;91(2):275-83.

17. Gogakos A, Barbetakis N, Lazaridis G, et al. Heimlich Valve and Pneumothorax. Ann Transl Med . 2015;3(4):4-8.

18. Heimlich HJ. Heimlich Valve for Chest Drainage. MedInstrum. 1983;17(1):29-31.

19. Paul AO, Kirchhoff C, Kay M V, et al. Malfunction of a Heimlich Flutter Valve Causing Tension Pneumothorax: Case Report of a Rare Complication. Patient Saf Surg. 2010;4(1):8. 